Iustitia Socialis. Revista Arbitrada de Ciencias Jurídicas.

Año IV. Vol. IV. Nº 1. Edición Especial 2019

Hecho el depósito de Ley: FA2016000064 ISSN: 2542-3371

FUNDACIÓN KOINONIA (F.K). Santa Ana de Coro, Venezuela

Clarisa Vélez

http://dx.doi.org/10.35381/racji.v4i1.548

\title{
El principio de territorialidad en la aplicación de las normas laborales de Ecuador y Venezuela
}

\section{The principle of territoriality in the application of labor standards of Ecuador and Venezuela}

\author{
Clarisa Vélez \\ clarisa abogada@hotmail.com \\ Universidad del Zulia, Maracaibo \\ Venezuela \\ https://orcid.org/0000-0002-3440-9272
}

Recibido: 30 de octubre de 2019

Aprobado: 03 de diciembre de 2019

\section{RESUMEN}

El objetivo general fue analizar el principio de territorialidad en la aplicación de las normas laborales de Ecuador y Venezuela, precisando la favorabilidad en el contexto de la globalización bajo el criterio interpretativo que propone el principio pro homine. Se justifica el estudio por la necesidad de reconocer estos criterios de validez espacial y temporal respecto de la aplicación de las normas laborales internacionales en la resolución de las controversias que se plantean con los trabajadores, mediante un estudio de tipo documental-jurídico, en un nivel analítico. Los resultados y las conclusiones apuntan hacia la reconsideración de la vigencia de los postulados clásicos, desde las aristas de la globalización, el uso de las nuevas tecnologías y el desarrollo de los derechos humanos, que ciertamente han permeado estos temas.

Descriptores: Derecho Laboral; derecho humanitario; derechos humanos; derecho internacional.

\section{ABSTRACT}

The general objective was to analyze the principle of territoriality in the application of the labor standards of Ecuador and Venezuela, specifying favorability in the context of globalization under the interpretative criteria proposed by the pro homine principle. The study is justified by the need to recognize these criteria of spatial and temporal validity regarding the application of international labor standards in the resolution of disputes that arise with workers, through a documentary-legal study, at a level analytical. The results and conclusions point to the reconsideration of the validity of the classical postulates, from the edges of globalization, the use of new technologies and the development of human rights, which have certainly permeated these issues. 
Descriptors: Labour law; humanitarian law; human rights; International law.

\section{INTRODUCCIÓN}

El principio de territorialidad en la aplicación de las normas laborales en Ecuador y Venezuela, es el objeto del estudio, el cual no puede ser examinado si no se atiende debidamente el contenido del principio de favorabilidad en el contexto de la globalización, ya que ambos son la base para la aplicación de las mismas, precisando que los nuevos contextos obligan a los intérpretes de la ley a reconocer el impacto de estos fenómenos en la sociedad moderna.

Hay temas que necesariamente deben pasar por el tamiz del desarrollo humano, de la evolución de los derechos fundamentales y de las nuevas tecnologías, enlazando este conocimiento con los postulados del Estado constitucional de derechos y de justicia, evitando que en el mundo real se reproduzcan relaciones asimétricas en todos los ámbitos, especialmente en el área laboral.

Queda claro, luego de la revisión documental que se ha explanado en este estudio, que la normativa internacional y la nacional que hoy regula la materia laboral, impone grandes desafíos, a quienes deben impartir justicia, ya que las clásicas nociones van perdiendo vigencia. La territorialidad y la favorabilidad, como conceptos preclaros que asientan la validez de las normas, están condenadas a ser exhaustivamente revisadas.

En este sentido, la relevancia del estudio estriba en el escrutinio de las modernas posturas que se asientan en esta materia, a partir de la redacción de textos como la Constitución de la República del Ecuador de 2008 y la Constitución de la República Bolivariana de Venezuela de 1999, especialmente de la configuración del derecho del trabajo como un hecho social, y de aquellos mecanismos dispuestos en las leyes especiales para garantizar el reconocimiento de las garantías que se disponen en favor del trabajador, en ambas naciones.

En el artículo se precisaron aspectos relativos al régimen laboral, nacional y extranjero, y la aplicación de las normas en la resolución de las controversias que se plantean con los trabajadores, analizando el contenido esencial del principio de 
territorialidad y favorabilidad en la doctrina del Ecuador y Venezuela, destacando la vigencia de estas instituciones jurídicas conforme evoluciona el fenómeno de la globalización, el uso de las nuevas tecnologías y el desarrollo de los derechos humanos, ya que ellos han transformado todo el mundo, buscando impactar positivamente en la vida de los ciudadanos.

En efecto, las preguntas que sirvieron al estudio son las siguientes: ¿Es posible la aplicación del derecho laboral extranjero en los conflictos que se planteen entre trabajadores y empleadores en la República del Ecuador y Venezuela?, y ¿En qué medida los clásicos principios de territorialidad y favorabilidad limitan o amplían el reconocimiento de las normas de derecho internacional en favor de los derechos de los trabajadores?

En consecuencia, fue menester traer a colación una serie de razonamientos vertidos en varios artículos científicos consultados, respecto de algunas correcciones que, en criterio de la investigadora, se han impuesto en la resolución de estos asuntos, entre estos el reconocimiento del principio pro homine, que allanó el camino hacia las conclusiones que se esbozaron al final del estudio.

Precisamente, se puede adelantar que la favorabilidad no puede hoy ser interpretada sino bajo las aristas del principio pro homine, que se impondrá en la resolución de conflictos de orden laboral, en el entendimiento de que es un atributo del ser humano, en razón de lo cual, debe pasarse aún por encima de criterios clásicos, como el de validez territorial de las normas, hallando en este un hilo conductor en la comprensión de nuevos significados. Para lo cual se tuvo como objetivo general analizar el principio de territorialidad en la aplicación de las normas laborales de Ecuador y Venezuela, precisando la favorabilidad en el contexto de la globalización bajo el criterio interpretativo que propone el principio pro homine. Se presentan fundamentos teóricos sustentadores de la investigación:

\section{Régimen Laboral, nacional y extranjero.}

El régimen laboral del Ecuador, en las últimas décadas, ha verificado una importante evolución y desarrollo, que le permiten situarse como una rama del derecho especialísima, con principios e instituciones propias, que centran la atención de 
cualquier estudioso de las ciencias jurídicas, respecto de la naturaleza del trabajo humano, ya no como una simple relación entre el trabajador y su empleador, sino como la expresión de la personalidad humana, en su dignidad y libertad, que debe ser reconocida y protegida por las leyes patrias, y así lo ha recogido el artículo 325 de la Constitución de la República de Ecuador (2008), cuando establece:

"El Estado garantizará el derecho al trabajo. Se reconocen todas las modalidades de trabajo, en relación de dependencia o autónomas, con inclusión de labores de autosustento y cuidado humano; y como actores sociales productivos, a todas las trabajadoras y trabajadores".

Desplegando una serie de principios en los dieciséis ordinales que conforman el artículo 326 eiusdem, que demarcan una serie de condiciones en materia laboral. Asimismo, el establecimiento del trabajo como un hecho social en Venezuela, ha tenido que transitar por diversas sendas, que han desembocado en su apreciación como una institución que está indisolublemente liada con el reconocimiento de la dignidad humana, y así aparece estipulado en los artículos 87, 88 y 89 de la Constitución de la República Bolivariana de Venezuela (1999). Es el artículo 89 del texto fundamental aprobado en Venezuela, el que consolida la relación laboral en los siguientes términos:

"El trabajo es un hecho social y gozará de la protección del Estado. La ley dispondrá lo necesario para mejorar las condiciones materiales, morales e intelectuales de los trabajadores y trabajadoras. Para el cumplimiento de esta obligación del Estado se establecen los siguientes principios: (...)

En este contexto surgen una serie de garantías mínimas que de acuerdo con la letra de los textos fundamentales que preceden, pueden resumirse en los siguientes principios: irrenunciabilidad de los derechos, indubio pro operario o favorabilidad, razonabilidad, buena fe, supremacía de la realidad sobre las formas o apariencias, intangibilidad y progresividad, igualdad y no discriminación, justicia social, que son de orden universal, en virtud del reconocimiento de las distintas recomendaciones y convenios que surgen de la Organización de Estados Americanos y la Organización de Naciones Unidas, en especial de la Organización Internacional del Trabajo, entre otras instancias.

Es así como la Organización Internacional del Trabajo (2010:22) señala expresamente: "todos los seres humanos, sin distinción de raza, credo o sexo, 
tienen derecho a perseguir su bienestar material y su desarrollo espiritual en condiciones de libertad y dignidad, de seguridad económica y en igualdad de oportunidades", claramente esta referencia se realiza en favor de los trabajadores y sus derechos.

Es a partir de la suscripción y ratificación, de los distintos cuerpos normativos que llegan a obtener un reconocimiento como fuentes normativas principales del derecho patrio, atendiendo a lo dispuesto a los artículos 425 y 22 de los textos fundamentales del Ecuador y Venezuela, respectivamente.

Estos principios han sido igualmente acogidos y desarrollados en el Código del Trabajo vigente en el Ecuador desde el año 2012 y la Ley Orgánica del Trabajo, de los Trabajadores y las Trabajadoras de Venezuela del año 2012, así como los distintos reglamentos y normas que rigen en la materia. En razón de lo cual es elemental entender que las viejas estructuras hayan cedido ante tal desarrollo que impide un trato que contraríe los más elementales derechos humanos que han sido reconocidos.

Ahora bien, son las relaciones asimétricas entre el trabajador y el empleador, las que han demandado una mayor atención por parte del Estado y de las organizaciones internacionales preenunciadas, quienes deben cumplir una función tuitiva del débil de la relación jurídica, como una demanda estricta y cierta de la consideración del trabajo como un derecho social y del principio de favorabilidad, en virtud del acaecimiento de la era que ha sido reconocida como la Constitucionalización de los Derechos Humanos, que impone una protección mucho más adecuada del problema planteado.

\section{Ámbito de aplicación de las normas en la resolución de las controversias que se plantean con los trabajadores.}

Cada uno de los instrumentos jurídicos nacionales y extranjeros, contienen claras normas para la regulación de las relaciones entre las partes involucradas en la actividad laboral, buscando, como lo explican Álvarez y Sánchez (2014), minimizar las desigualdades legales en función de lograr la aplicación de la justicia social, es allí, donde los principios señalados, adquieren su verdadero valor, lo que impone un 
acercamiento a los criterios de validez espacial, como el de territorialidad y validez temporal, como la favorabilidad en los términos que siguen.

Canessa (2008), en este sentido expone que los derechos humanos laborales reconocen como único titular universal a la persona, con el respeto de su dignidad humana y satisfaciendo sus necesidades básicas en el ámbito laboral, en razón de esto, el principio de territorialidad se alza como necesario para la discusión que se pretende en esta materia.

2.1. El principio de territorialidad en la ley y doctrina del Ecuador y Venezuela.

En el Ecuador, el Código del Trabajo en su artículo 1 dispone el ámbito de aplicación de la ley, estableciendo que será dicha norma la que regule las relaciones entre los empleadores y trabajadores, sin importar la nacionalidad de los intervinientes, apelando al criterio territorial que se aplica por el simple hecho de que la suscripción del contrato individual, tal y como lo dispone el artículo 8, eiusdem se ha realizado en el espacio geográfico de la República.

Respecto a la norma que regula la aplicación de la ley laboral en el espacio venezolano, el artículo 3 de la Ley Orgánica del Trabajo, los Trabajadores y las Trabajadoras (2012), especifica el principio de territorialidad, el cual se impone como un criterio fundamental para la regulación de las relaciones y eventuales conflictos laborales que se presenten en el ámbito geográfico venezolano, derivados de la visión del trabajo como un hecho social.

Para Platán (2015), en un sentido genérico, el ámbito espacial de validez de una norma jurídica, está referido a la porción de espacio en que un precepto jurídico es aplicable, es decir, a la porción de terreno, de espacio-territorio donde se pueda aplicar una determinada ley, en otras palabras, dónde tendrá eficacia jurídica.

De igual modo, se observa que, por disposición constitucional, en ambas naciones se reconoce la aplicación de los instrumentos internacionales en la regulación de estas actividades, dando paso al derecho extranjero en la resolución de conflictos que puedan derivarse de la misma, basándose en el criterio clásico de favorabilidad o indubio pro operario, que, en criterio de la autora, queda hoy supeditado al reconocimiento del principio pro homine o pro persona. 
En este escenario, es menester hacer una lacónica referencia al principio pro homine, ya que se encuentra relacionado con el impacto que tiene la era de la Constitucionalización de los Derechos Humanos en las variadas disciplinas del conocimiento, aspecto que se encuentra indisolublemente ligado al fenómeno de la globalización. En la línea de pensamiento descrito, se debe advertir que la globalización es un detonador del desarrollo de los Derechos Humanos, por lo que Stiglitz (2002: 37), es enfático al afirmar que la globalización, es:

"la integración más estrecha de los países y los pueblos del mundo, producida por la enorme reducción de los costes de transporte y comunicación, y el desmantelamiento de las barreras artificiales a los flujos de bienes, servicios, capitales, conocimientos y (en menor grado) personas a través de las fronteras. La globalización ha sido acompañada por la creación de nuevas instituciones; en el campo de la sociedad civil internacional...".

En particular, en el derecho laboral, la globalización ha generado claras consecuencias a partir de los acontecimientos que se sucedieron con la Revolución Industrial, lo cual trajo aparejada distintas manifestaciones como las protestas, la huelga y la sindicalización de los trabajadores, que han decisivamente han demarcado la actuación del Estado en esta materia, cuya evolución pasa desde la no injerencia en estos asuntos hasta el carácter tuitivo del mismo.

La influencia que dicho proceso tiene sobre el orden jurídico patrio y extranjero, recae en temas tan trascendentales como el de la soberanía, y particularmente resuena sobre el ámbito espacial de aplicación de las normas laborales, sobre todo cuando se atiende al contraste con el reconocimiento del Derecho Extranjero en la resolución de las controversias que se susciten entre empleador y trabajadores.

En efecto, la soberanía ha sido el principal atributo de la nación, que ha forjado algunos cuestionamientos respecto de la vigencia de los contenidos en los instrumentos de orden internacional al interior de las Repúblicas, por lo que algunos doctrinarios, entre estos Becerra, Povedano y Tellez (S/F: 23), incluso llegan a afirmar, que: "el concepto de soberanía ya no existe en la actualidad, que no se puede hablar de soberanía si estamos frente a la unión de Estados soberanos que aceptan ceder esa soberanía en pro de la Unión". 
Como claro ejemplo de esto, oponen los autores la aparición de la Unión Europea, respecto de la cual ilustran que ya no se puede hablar de la clásica soberanía, pues estas naciones, han sometido su libertad e independencia en pro de alcanzar fines comunes, entre estas basta destacar el área económica, explican que, hoy día, se apuesta por la creación de entes supraestatales y de la promulgación de una Constitución europea, con lo cual se acabaría con el esquema antiguo de que la nación es la que detenta la soberanía.

Sin embargo, algunos mantienen su postura respecto de la pertinencia actual del término soberanía, bajo la vertiente clásica, y otros, sencillamente, llegan a afirmar que lo que ha ocurrido es una verdadera evolución de los conceptos que están liados con la soberanía, que permite a los Estados concertar acciones comunes para atender de manera eficaz y eficiente las demandas de la población, verbigracia, el tema del crimen internacional, de la propia consolidación y vigencia de los derechos humanos, de la economía internacional, entre otros, lo que ciertamente conduce a un replanteamiento de los atributos de la soberanía, como son: el territorio, el pueblo y el gobierno, discusión que pone en la mesa aquellos asuntos relacionados con las controversias de tipo laboral y las posibles y plausibles soluciones que impone esta nueva era.

En el tema que interesa, se infiere conforme los autores supra citados, una vez que realizan una disertación sobre temas claves relacionados con los atributos de la soberanía, que dicho concepto "medieval", no ha desaparecido solo que: "se ha transformado dramáticamente, al parejo de las relaciones internacionales del siglo $X X I$, que están caracterizadas por un desarrollo impresionante de la sociedad que sigue las pautas de la III revolución industrial" (Becerra, Povedano y Tellez, S/F: 81).

\section{El principio de favorabilidad en la doctrina del Ecuador y Venezuela.}

En contraste con la regulación interna, los dispositivos protectores de la libertad contenidos en los instrumentos internacionales de Derechos Humanos, suscritos por el Ecuador y Venezuela, se incorporan en cada una de las constituciones, verbigracia la previsión contenida en los artículos 425 (Ecuador) y 23 (Venezuela). Así las cosas, el artículo 425 de la Constitución del Ecuador dispone: 
"El orden jerárquico de aplicación de las normas será el siguiente: La Constitución; los tratados y convenios internacionales; las leyes orgánicas; las leyes ordinarias; las normas regionales y las ordenanzas distritales; los decretos y reglamentos; las ordenanzas; los acuerdos y las resoluciones; y los demás actos y decisiones de los poderes públicos.

En caso de conflicto entre normas de distinta jerarquía, la Corte Constitucional, las juezas y jueces, autoridades administrativas y servidoras y servidores públicos, lo resolverán mediante la aplicación de la norma jerárquica superior.

La jerarquía normativa considerará, en lo que corresponda, el principio de competencia, en especial la titularidad de las competencias exclusivas de los gobiernos autónomos descentralizados".

Igualmente, el artículo 22 de la Constitución de la República Bolivariana de Venezuela, a la letra señala: "La enunciación de los derechos y garantías contenidas en esta Constitución no debe entenderse como negación de otros que, siendo inherentes a la persona humana, no figuren expresamente en ella", amplificando de este modo su espectro de actuación, bajo la concepción del principio pro homine, que como ilustra Pinto (2014:9), es:

"un criterio hermenéutico que informa todo el derecho de los derechos humanos, en virtud del cual se debe acudir a la norma más amplia, o a la interpretación más extensiva, cuando se trata de reconocer derechos protegidos e, inversamente, a la norma o a la interpretación más restringida cuando se trata de establecer restricciones permanentes al ejercicio de los derechos o su suspensión extraordinaria. Este principio coincide con el rasgo fundamental del derecho de los derechos humanos, esto es, estar siempre a favor del hombre".

De acuerdo con esto, la favorabilidad como principio ocupa un lugar privilegiado en los textos fundamentales de ambas naciones, ubicándose como un criterio que es necesario para la resolución de las controversias planteadas en el ámbito laboral.

3. Vigencia del principio de territorialidad y favorabilidad bajo las aristas de la globalización, el uso de las nuevas tecnologías y el desarrollo de los derechos humanos.

Es claro advertir que es a partir de la segunda mitad del siglo XIX, que el derecho laboral cuenta con un respaldo internacional que deviene de la conformación de la Organización Internacional del Trabajo, que está encargada de la creación de convenios y distintas regulaciones del derecho laboral individual o colectivo. 
La globalización, genera profundas modificaciones del orden jurídico patrio, que no puede pensarse se circunscriban únicamente al ámbito económico, también ha llegado a influir en el campo del derecho laboral, derecho penal, del derecho ambiental, del derecho de familia, por solo nombrar algunos, lo que llama la atención sobre el diseño de una norma común que impida las desventajas o efectos negativos que, en ocasiones, trae aparejado el proceso de globalización.

Así la función del derecho bajo este entramado de cosas, es brindar la seguridad jurídica necesaria a los intervinientes para desarrollar cada una de sus actividades dentro del margen del respeto por los derechos humanos fundamentales, que de igual modo han sido una conquista de la humanidad toda, que reposa en los valores de libertad, justicia e igualdad.

En consecuencia, tal y como lo ilustra Carbonell (2004), es imperioso rescatar el papel del sistema jurídico internacional y la cooperación entre las naciones para enfrentar las amenazas concretas sobre estos bienes e intereses, sobre todo considerando que el derecho laboral es una ciencia con un amplio desarrollo humanista por los fines que persigue su regulación.

En correspondencia con lo delatado, se precisa que, la vigencia de los criterios expuestos, están condicionados al replanteo de los términos ley, soberanía y economía, en un mundo global, ya que como advierten los autores consultados, la globalización, con sus ventajas y desventajas, llegó para quedarse, en consecuencia se requiere un alto grado de consciencia y creatividad para enfrentar los retos y desafíos que impone dicho proceso, evitando reducir el problema al campo de lo puramente económico, donde el fenómeno muestra su rostro más grotesco, entendiendo que: "la ley del más fuerte se impone ante la ausencia de reglas de alcance planetario para dominar a los emergentes poderes económicos." (Ferrajoli, citado por Carbonell, 2004)

Son mucho los retos que le corresponde afrontar al Ecuador y a Venezuela ante el mundo globalizado, atendiendo siempre a la agitada agenda política, social, económica, y de cualquier otra índole, que afrontan hoy día estos países, entre estos destaca la ausencia de un arquetipo legal que de manera eficaz genere claras reglas para todos los involucrados en los asuntos de orden laboral. 
Ahora bien, se apela al entendimiento y a la racionalidad de las respectivas Cortes Constitucionales, donde las juezas y jueces, autoridades administrativas y servidoras y servidores públicos, quienes, bajo el ejercicio de la soberanía, tendrán la última palabra, y a la doctrina del precedente, para evitar en caso de conflictos potenciales relacionados con los temas expuestos, el desconocimiento de las libertades fundamentales alcanzadas e incorporadas en cada una de las normas jurídicas de carácter universal y de la propia Constitución, atendiendo a la ley, y a los principios de orden laboral, como verdaderos diques de contención ante el ejercicio arbitrario del poder.

Como se puede observar, el principio constitucional de la democracia tiene vigencia en el campo económico, administrativo, cultural, social, educativo, sindical o gremial del país, y en algunos aspectos de la vida privada de las personas; y su objetivo primordial es el de posibilitar y estimular la intervención de los ciudadanos en actividades relacionadas con la gestión pública y en todos aquellos procesos decisorios incidentes en la vida y en la orientación del Estado y de la sociedad civil (Bobbio, 1981), de lo cual no queda más que aceptar su aplicación en materia laboral.

Es fácil colegir que, una de las intencionalidades más claras de la Constitución de la República del Ecuador 2008, y de la Constitución de la República Bolivariana de Venezuela de 1999, fue la de iniciar un proceso de complementar la democracia con el verdadero reconocimiento de los Derechos Humanos Fundamentales. Dichas Cartas Políticas, definen los ámbitos de validez de las leyes, para el ejercicio de esta nueva forma de organización, que, en ambos casos, llegan a estructurarse con base en cinco poderes, abogando en todo momento por una participación de los ciudadanos en estos quehaceres, para el logro de los objetivos propuestos.

Ante los desafíos delatados, se ha llegado a ensayar algunas soluciones, entre estas, rescatar el rol del sistema jurídico internacional, apelar a la cooperación entre las naciones, la existencia de un espacio público multilateral, y auspiciar de manera decidida los movimientos sociales, incluso se llega a considerar como lo reseña Carbonell (2004), la creación de un constitucionalismo supra-nacional. Será cuestión de tiempo, para ver si se consolidan estos planteamientos de raigambre liberal. 
Iustitia Socialis. Revista Arbitrada de Ciencias Jurídicas.

Año IV. Vol. IV. № 1. Edición Especial 2019

Hecho el depósito de Ley: FA2016000064 ISSN: 2542-3371

FUNDACIÓN KOINONIA (F.K). Santa Ana de Coro, Venezuela

Clarisa Vélez

\section{MÉTODO}

La metodología empleada para la edificación de la investigación, se basó en una tipología documental - bibliográfica, sostenida desde la perspectiva de Palella Stracuzzi y Martins Pestana (2012), lo que permitió analizar teorías, leyes, procedimientos judiciales, jurisprudencias, bibliografías, como parte de la población de estudio, generándose mediante el análisis documental jurídico, la construcción de aportes reflexivos teóricos en el campo del derecho internacional.

\section{RESULTADOS}

El primer aspecto a considerar en el desarrollo del estudio fue el relativo al Régimen Laboral, nacional y extranjero, para lo cual se abordó la evolución y desarrollo que se ha experimentado en el Ecuador y Venezuela en esta materia, destacando que el reconocimiento de los principios e instituciones propias, respecto de la naturaleza del trabajo humano, permiten concebir a este hecho social, ya no como una simple relación entre el trabajador y su empleador, sino como la expresión de la personalidad humana, en su dignidad y libertad, como lo han dispuesto los artículos 325 y 326 de la Constitución de la República de Ecuador (2008), y los artículos 87, 88 y 89 de la Constitución de la República Bolivariana de Venezuela (1999).

Se consolida así un conjunto normativo que, en todo momento propenderá a la protección del trabajador, como especial sujeto de esta relación, e instituciones que atenderán, con prioridad absoluta, las demandas legítimas de los trabajadores, entre estas se ubica la Organización Internacional del Trabajo, que ha tenido como norte la resolución de conflictos, asegurando los propósitos comunes que ha fijado el orbe en esta materia, es así como el reconocimiento de los derechos de los trabajadores, encuentra un campo fértil en el ámbito internacional y patrio, a partir de la suscripción y ratificación, de los distintos instrumentos, que como ha quedado señalado, son fuentes directas en el ámbito laboral.

En este estado de cosas, Álvarez y Sánchez (2014), advierten algunos nudos problemáticos que aun requieren de una mayor atención del Estado, estos son los conflictos que se suscitan a partir de las relaciones asimétricas entre el trabajador y 
el empleador, que pondría en tela de juicio la consideración del trabajo como un derecho social, y la dignidad y la libertad del trabajador como el derrotero a seguir. Conforme con lo expuesto, el segundo de los objetivos trazados, fue el de la determinación del ámbito de aplicación de las normas en la resolución de las controversias que se plantean con los trabajadores, para lo cual Álvarez y Sánchez (2014), precisan que cualquier tipo de conflicto que se presente en esta área, debe ser atendida conforme lo precisan las normas patrias e internacionales, evitando las profundas desigualdades que se han evidenciado en la historia, pasada y reciente, y que obstaculizan la justicia social.

Se impone, como necesario atender a los criterios de validez espacial, como el de territorialidad y validez temporal, como la favorabilidad, entendiendo como expresa Canessa (2008), que los derechos humanos laborales reconocen como único titular universal a la persona, independientemente de donde se encuentre situado el trabajador o cual sea la ley vigente, hoy día no se puede aludir al concepto de territorio, ni siquiera al de vigencia de la norma, como se ha hecho en otros tiempos, ya que la era de la globalización, ha venido para quedarse, y se imponen hoy con más rigor que nunca, leyes comunes a todas las naciones, y el principio de favorabilidad ante la ley, que a nuestro criterio, ha sido cobijado por el principio pro homine, que se convierte en un principio de interpretación transversal de todo el ordenamiento jurídico.

A pesar de lo expuesto, se encuentra que, ante cada conflicto que se presenta en la materia, se alude inmediatamente a lo dispuesto en el artículo 1 del Código del Trabajo del Ecuador o al artículo 3 de la Ley Orgánica del Trabajo, los Trabajadores y las Trabajadoras de Venezuela, que dispone el ámbito de aplicación de la ley bajo el criterio territorial que, encuentra su justificación en el hecho de que la suscripción del contrato se ha realizado en el espacio geográfico de la República.

Se discute bajo lo planteado con antelación, lo que ilustra Platán (2015), al advertir que el ámbito espacial de validez de una norma jurídica, está referido a la porción de espacio en que un precepto jurídico es aplicable o dónde este tiene eficacia jurídica, ya que se insiste, la globalización, ha permitido una flexibilización de estos términos, sobre todo a partir del reconocimiento constitucional, en ambas naciones, de la 
aplicación de los instrumentos internacionales y del reconocimiento del principio pro homine o pro persona.

El desarrollo de los Derechos Humanos, ha tenido como detonante principal la globalización, llevando a Stiglitz (2002), a afirmar que este fenómeno ha supuesto la creación de nuevas instituciones, entre estas, aparecen normas que buscan reivindicar las prerrogativas alcanzadas por los trabajadores, en sus incesantes luchas, donde se articulan derechos fundamentales como la huelga y la sindicalización.

Se une a este espectro de cambios o transformaciones, la consideración de temas como el de soberanía del Estado, en razón de que aquí se ubica el sustrato de aplicación de las normas patrias, que toman como base el territorio, sin embargo, Becerra, Povedano y Tellez (S/F), ya explican que no hay tal atributo, cuando se habla por ejemplo de la Unión Europea, y de sus fines comunes.

Iguales fines, se intentan propiciar o conseguir, a partir de la consolidación de normas o entes supraestatales que atiendan las legítimas demandas de los trabajadores, y así lo ha reconocido cada Estado parte de la comunidad internacional, en sus propios textos fundamentales, apostando por la vigencia de los derechos humanos, que no se puede negar han tenido una evolución descollante en los últimos siglos.

El tercer objetivo propuesto, lleva al reconocimiento del principio de favorabilidad del trabajador, el cual se impone, en la legislación de Ecuador y de Venezuela, conforme se ha perfilado en los instrumentos internacionales de Derechos Humanos, que son parte de la estructura normativa interna.

De igual modo, el principio de favorabilidad se ha dispuesto en la doctrina como un principio de interpretación que según refiere Pinto (2014), alude a los resultados de este proceso hermenéutico, restrictivo o extensivo, siempre bajo la consideración de aquello que más favorezca al trabajador, rescatando del autor, la aseveración que lo lleva a considerar que este criterio: "coincide con el rasgo fundamental del derecho de los derechos humanos, esto es, estar siempre a favor del hombre", en la resolución de las controversias planteadas en el ámbito laboral, especialmente en aquellas relaciones de tipo asimétrico entre trabajador y patrono. 
Como corolario de lo expuesto, se entiende que hay una relación disfuncional entre la validez espacial y la temporal como está planteado desde la normativa interna laboral de países como Ecuador y Venezuela, con la vigencia del principio de territorialidad y favorabilidad bajo las aristas de la globalización, el uso de las nuevas tecnologías y el desarrollo de los derechos humanos.

Se advierte claramente que el derecho laboral cuenta con un respaldo internacional que traspasa fronteras, que hay un objetivo común en los países que conforman la comunidad de naciones, respecto del reconocimiento de la dignidad y libertad de los trabajadores, ubicando su labor como un hecho social que le permite desarrollarse como persona, en igualdad de oportunidades y con seguridad jurídica, dentro del margen del respeto por los derechos humanos fundamentales.

Pero no solo debe quedarse este reconocimiento en el plano normativo, es necesario, que basados hoy en la era de la globalización, el uso de las nuevas tecnologías y el desarrollo de los derechos humanos, combatir certeramente las amenazas que se ciernen sobre los derechos de los trabajadores, que en última instancia afectan su dignidad.

Las acciones que se adopten en esta materia en el ámbito internacional deben ensayarse en el ámbito nacional, ya que la jurisprudencia en materia laboral, no debe circunscribirse a un solo espacio territorial, en un mundo globalizado. En este sentido, se deben ensayar soluciones comunes a problemas comunes, donde se deben instar al espíritu reivindicativo que signó las luchas propias de la revolución industrial, y colocarla en cabeza de los ciudadanos, como principales defensores de estas conquistas.

\section{CONCLUSIONES}

A pesar de que se han asentado criterios como el de territorialidad y favorabilidad respecto de la aplicación e interpretación de las normas laborales, en relación con las consecuencias que se derivan del fenómeno de la globalización, las nuevas tecnologías y el desarrollo de los derechos humanos, Ecuador y Venezuela enfrentan serios retos, verdaderas pruebas de fuego, ante los conflictos que produce la alianza: territorialidad-favorabilidad-derechos humanos, que necesariamente 
deben ser objeto de algunos replanteamientos ante la democracia y el reconocimiento del propio Estado constitucional de derechos y de justicia, recurriendo a la ley (nacional o internacional) como límite ante los abusos que pueden generarse producto de las relaciones asimétricas que están vigentes en este ámbito.

Pese a la vigencia del principio de territorialidad, el desarrollo de una amplia plataforma normativa internacional, impone como otro desafío, la creación de un modelo legal que permita reconducir las respuestas ante los conflictos que se presenten entre las partes que se ubican bajo las condiciones del contrato individual, generando seguridad jurídica para todos los involucrados.

En esta línea argumentativa aparece Strange (2001:7), quien simplemente advierte que: "las fronteras territoriales ya no coinciden con los límites que la autoridad política mantiene sobre la economía y la sociedad", aduciendo que estos acontecimientos invitan a repensar las instituciones jurídicas, aun aquellas de mayor abolengo.

De esta cita es fácil inferir que el proceso de globalización haya dado al traste con el término Estado-Nación, que necesariamente debe evolucionar hasta adaptarse a los profundos cambios que trae aparejada la proliferación de eventos de orden mundial, como la aparición de las nuevas tecnologías, del flujo de comunicaciones, y del comercio internacional, sin necesidad de negar la existencia del mismo, agregando a este comentario, el evidente impacto que sufre el principio de territorialidad como pilar de aplicación de las leyes nacionales.

Ahora bien, hay que tener conciencia de que el principio de territorialidad no desaparecerá de los textos normativos patrios, como parte importante del ejercicio de la soberanía, acogiendo la corriente o vertiente "transformacionalista" según la cual el Estado (con sus principales atributos) no está desapareciendo ni debilitándose, como se suele pensar, sólo se están produciendo cambios a nivel de sus roles y funciones, y así lo explica Romero (2008), al indicar que los Estados nacionales, están pasando por un proceso de adaptación y transformación, sin que esto implique la pérdida de su soberanía. 
De acuerdo con esto, es sensato asumir que cada nación, incluyendo Ecuador y Venezuela, se han visto fuertemente influenciadas en el ejercicio de su poder por la globalización, las nuevas tecnologías y el desarrollo de los derechos humanos, sin que pueda esto llegar a considerarse una renuncia de las funciones que deben ejercer dentro de sus propios límites fronterizos, o un debilitamiento de su poder.

Bajo esta postura señala Sassen (2007), los Estados se adaptan y transforman, en efecto, la autoridad estatal se desnacionaliza y se produce un desplazamiento de las funciones de gobernabilidad pública hacia otras instancias, nacionales o supranacionales, sin que esto pueda considerarse un obstáculo para el ejercicio de la soberanía.

Se llega a señalar incluso la existencia de un Estado transnacional, que debe ampliar sus funciones para adaptarse a los cambios que supone el mundo globalizado, que de manera intempestiva llego para quedarse.

Atendiendo a la soberanía y sus atributos, será necesario examinar el principio de territorialidad como criterio de aplicación e interpretación de la ley laboral, para lograr una mayor utilidad del mismo en este mundo globalizado. Destacando en este particular el papel que debe desempeñar el principio de favorabilidad, que ha quedado supeditado al reconocimiento del principio pro homine, que ubica a los trabajadores como portadores de determinados intereses sociales, y que apuntan al beneficio colectivo, de ahí la importancia de concebir al trabajo como un hecho social.

En síntesis, será tarea primordial del Derecho, en general, buscar una salida a las controversias que se producen cuando se lían estos fenómenos, procurando un orden mundial justo, tal y como lo refiere Alegría (S/F).

De lo expuesto, queda claro que, la doctrina laboral de corte garantista, conjuntamente con las normas del constitucionalismo moderno, rechazan de plano los abusos, arbitrariedades y atropellos contra el ciudadano y sus derechos, que socavan las bases mismas de la sociedad o el status ético - jurídico, encontrándose aquí la ratio essendi para el reconocimiento de los derechos y libertades fundamentales que han marcado la historia mundial.

En este aserto, señala Sánchez (2012), que para aproximarse a una correcta 
comprensión del principio de favorabilidad o indubio pro operario, sustantivo y adjetivo, se debe tener en cuenta que en la base de su formación tiene lugar un conflicto entre dos tendencias que normalmente han sido presentadas como antagónicas (trabajador y empleador) y cuya resolución pasa por el tamiz del reconocimiento pleno de los derechos humanos que subyacen hoy día bajo la consideración del principio pro homine.

Esta visión ha estructurado las distintas respuestas que han ensayado cada una de estas naciones a lo largo de la historia, y será precisamente el análisis filosóficojurídico sobre el principio pro homine, el que permitirá hoy día reconocer la vigencia del derecho extranjero en la resolución de conflictos de orden laboral cuando desde esta plataforma se adviertan mejores condiciones que las dispuestas en el contrato individual, esta posición que se asume como respuesta a la incógnita planteada, no escapará de algunas críticas y oposiciones apelando a la corriente clásica que ubica el principio de territorialidad como fundamento de aplicación del derecho interno, por lo que también resultará útil su reconocimiento para hallar un hilo conductor en la comprensión de sus significados.

Por otra parte, es un hecho cierto que el fenómeno de la globalización requiere el replanteamiento de los roles y funciones del Estado, pero no por esto se puede inferir que el Estado, con sus atributos esenciales se encuentre debilitado, sólo se trata de replantear aquellas concepciones tradicionales, como la soberanía, entendida esta como un poder absoluto, la democracia, y los modelos políticos adoptados por cada país, que obviamente se han ido desdibujando bajo las formas modernas de convivencia internacional con otros países de la comunidad, se trata pues de alertar sobre las transformaciones que han resultado patentes en este proceso, las cuales deben ser objeto de puntual estudio, a fin de comprender hoy su verdadera esencia y alcance. 
Iustitia Socialis. Revista Arbitrada de Ciencias Jurídicas.

Año IV. Vol. IV. Nº 1. Edición Especial 2019

Hecho el depósito de Ley: FA2016000064 ISSN: 2542-3371

FUNDACIÓN KOINONIA (F.K). Santa Ana de Coro, Venezuela

Clarisa Vélez

\section{REFERENCIAS CONSULTADAS}

1. Alegría, Héctor $(S / F)$. Globalización y Derecho. Recuperado a partir de www.derecho.uba.ar/publicaciones/pensar-en-derecho/.../globalizacion-yderecho.pdf.

2. Álvarez, Rebeca y Sánchez, Roslyn (2014). Principios fundamentales del derecho procesal laboral venezolano. ANUARIO. Volumen 37, Año 2014, pp. 109-132.

3. Asamblea Constituyente de la República Bolivariana de Venezuela (1999). Constitución de la República Bolivariana de Venezuela. Caracas, Venezuela. Gaceta Oficial de la República Bolivariana de Venezuela No. 36.860. 30 de diciembre de 1999.

4. Asamblea Constituyente de la República del Ecuador. (2008). Constitución de la República del Ecuador. Quito, Ecuador. Decreto Legislativo 0. Registro Oficial 449 del 20 de octubre de 2008.

5. Asamblea Nacional de la República Bolivariana de Venezuela (2012). Ley Orgánica del Trabajo, de los Trabajadores y las Trabajadoras. Caracas, Venezuela. Gaceta Oficial de la República Bolivariana de Venezuela Extraordinaria No. 6.076 del 07 de mayo de 2012.

6. Asamblea Nacional de la República del Ecuador. (2012). Código del Trabajo. Quito, Ecuador. Registro Oficial Suplemento 167 del 16 de diciembre de 2005. Última modificación 26 de septiembre de 2012. Codificación 2005-17. Estado: vigente.

7. Becerra, Manuel; Povedano, Adriana y Tellez, Evelyn (S/F). La soberanía en la era de la globalización. Recuperado a partir de www. www.juridicas.unam.mx.

8. Bobbio, Norberto (1981). Participación Política. Diccionario de Política, Siglo XXI Editores. México.

9. Canessa, M. (2008). Los derechos humanos laborales: el núcleo duro de derechos "core right" y el "ius cogens" laboral. Revista del Ministerio de Trabajo e Inmigración, número 72, pp. 111-151.

10. Carbonell, Miguel (2004). Constitucionalismo y Democracia. Ensayos Críticos, Porrua, México.

11. Organización Internacional del Trabajo (2010). Constitución de la Organización Internacional del Trabajo y textos seleccionados. Oficina Internacional del Trabajo. Ginebra, Suiza. 
12. Patlán Pérez, Juana (2016). "Derechos laborales: una mirada al derecho a la calidad de vida en el trabajo". En: Ciencia Ergo Sum, Volumen 23, N²-2016. Universidad Autónoma del Estado de México. Recuperado a partir de http://cienciaergosum.uaemex.mx.

13. Palella Stracuzzi, S. \& Martins Pestana, F. (2012). Metodología de la investigación cuantitativa. Fondo editorial de la Universidad Pedagógica Libertador. Caracas, Venezuela.

14. Pinto, Mónica (2014). "El principio pro homine. Criterios de hermenéutica y pautas para la regulación de los derechos humanos". Buenos Aires, Argentina, pp. 1-19. Recuperado a partir de www.derechoshumanos.unlp.edu.ar/.../el-principio-pro-homine-criterios-dehermeneutica-y-pautas-para-la-regulacion-de-los-derechos-humanos.pdf.

15. Romero, Inés (2008). El debate sobre la globalización. En: Programa de Formación. Lima, Perú.

16. Sánchez Sorondo, Marcelo (2007). “¿El ocaso de las naciones?”. El Estado, en la Aldea Global, 8vas Jornadas Bancarias de la República Argentina, ABRA, julio de 2007. Buenos Aires, Argentina.

17.Sassen, Saskia (2007). Una Sociología de la Globalización. Katz Editores, Buenos Aires, Argentina.

18. Stiglitz, Joseph (2002). El malestar en la globalización, Taurus, Buenos Aires, Argentina.

19. Strange, Susan (2001). La retirada del Estado. La difusión del poder en la economía mundial. Icaria editorial - IntermónOxfam, Barcelona, España.

\section{REFERENCES CONSULTED}

1. Joy, Hector (S / F). Globalization and Law. Recovered from www.derecho.uba.ar/publicaciones/pensar-en-derecho/.../globalizacion-yderecho.pdf.

2. Álvarez, Rebeca and Sánchez, Roslyn (2014). Fundamental principles of Venezuelan labor procedural law. YEARBOOK. Volume 37, Year 2014, pp. 109-132.

3. Constituent Assembly of the Bolivarian Republic of Venezuela (1999). Constitution of the Bolivarian Republic of Venezuela. Caracas Venezuela. Official Gazette of the Bolivarian Republic of Venezuela No. 36,860. December 30, 1999. 
Iustitia Socialis. Revista Arbitrada de Ciencias Jurídicas.

Año IV. Vol. IV. N 1 . Edición Especial 2019

Hecho el depósito de Ley: FA2016000064 ISSN: 2542-3371

FUNDACIÓN KOINONIA (F.K). Santa Ana de Coro, Venezuela

Clarisa Vélez

4. Constituent Assembly of the Republic of Ecuador. (2008). Constitution of the Republic of Ecuador. Quito, Ecuador. Legislative Decree 0. Official Record 449 of October 20, 2008.

5. National Assembly of the Bolivarian Republic of Venezuela (2012). Organic Law of Labor, Workers and Workers. Caracas Venezuela. Official Gazette of the Bolivarian Republic of Venezuela Extraordinary No. 6,076 of May 7, 2012.

6. National Assembly of the Republic of Ecuador. (2012). Work code. Quito, Ecuador. Official Registry Supplement 167 of December 16, 2005. Last modified September 26, 2012. Codification 2005-17. Status: current.

7. Becerra, Manuel; Povedano, Adriana and Tellez, Evelyn (S / F). The sovereignty in the globalization era. Recovered from www. www.juridicas.unam.mx.

8. Bobbio, Norberto (1981). Political participation. Dictionary of Politics, 21st Century Publishers. Mexico.

9. Canessa, M. (2008). Labor human rights: the hard core of rights "core right" and the "ius cogens" labor. Magazine of the Ministry of Labor and Immigration, number 72, pp. 111-151.

10. Carbonell, Miguel (2004). Constitutionalism and Democracy. Critical Essays, Porrua, Mexico.

11. International Labor Organization (2010). Constitution of the International Labor Organization and selected texts. International Labor Office. Geneva, Switzerland.

12. Patlán Pérez, Juana (2016). "Labor rights: a look at the right to quality of life at work". In: Ergo Sum Science, Volume 23, No. 2-2016. Autonomous Mexico State University. Recovered from http://cienciaergosum.uaemex.mx.

13. Palella Stracuzzi, S. \& Martins Pestana, F. (2012). Quantitative research methodology. Editorial Fund of the Libertador Pedagogical University. Caracas Venezuela.

14. Pinto, Monica (2014). "The pro homine principle. Hermeneutic criteria and guidelines for the regulation of human rights ". Buenos Aires, Argentina, pp. 119. Recovered from www.derechoshumanos.unlp.edu.ar/.../el-principio-prohomine-criterios-de-hermeneutica-y-pautas-para-la-regulacion-de-losderechos-humanos. pdf.

15. Romero, Inés (2008). The debate on globalization. In: Training Program. Lima Peru. 
16. Sánchez Sorondo, Marcelo (2007). "The twilight of the nations?" The State, in the Global Village, 8th Banking Days of the Argentine Republic, ABRA, July 2007. Buenos Aires, Argentina.

17. Sassen, Saskia (2007). A sociology of globalization. Katz Editores, Buenos Aires, Argentina.

18. Stiglitz, Joseph (2002). The malaise in globalization, Taurus, Buenos Aires, Argentina.

19. Strange, Susan (2001). The withdrawal of the State. The diffusion of power in the world economy. Icaria editorial - IntermónOxfam, Barcelona, spain. 